\title{
Research Article \\ Stable Dyonic Thin-Shell Wormholes in Low-Energy String Theory
}

\author{
Ali Övgün ${ }^{1,2}$ and Kimet Jusufi ${ }^{3,4}$ \\ ${ }^{1}$ Instituto de Física, Pontificia Universidad Católica de Valparaíso, Casilla 4950, Valparaíso, Chile \\ ${ }^{2}$ Physics Department, Arts and Sciences Faculty, Eastern Mediterranean University, Famagusta, Northern Cyprus, Mersin 10, Turkey \\ ${ }^{3}$ Physics Department, State University of Tetovo, Ilinden Street nn, 1200 Tetovo, Macedonia \\ ${ }^{4}$ Institute of Physics, Faculty of Natural Sciences and Mathematics, Ss. Cyril and Methodius University of Skopje, \\ Arhimedova 3, 1000 Skopje, Macedonia
}

Correspondence should be addressed to Kimet Jusufi; kimet.jusufi@unite.edu.mk

Received 22 June 2017; Revised 15 September 2017; Accepted 3 October 2017; Published 1 November 2017

Academic Editor: George Siopsis

Copyright (C) 2017 Ali Övgün and Kimet Jusufi. This is an open access article distributed under the Creative Commons Attribution License, which permits unrestricted use, distribution, and reproduction in any medium, provided the original work is properly cited. The publication of this article was funded by SCOAP ${ }^{3}$.

Considerable attention has been devoted to the wormhole physics in the past 30 years by exploring the possibilities of finding traversable wormholes without the need for exotic matter. In particular, the thin-shell wormhole formalism has been widely investigated by exploiting the cut-and-paste technique to merge two space-time regions and to research the stability of these wormholes developed by Visser. This method helps us to minimize the amount of the exotic matter. In this paper, we construct a four-dimensional, spherically symmetric, dyonic thin-shell wormhole with electric charge $Q$, magnetic charge $P$, and dilaton charge $\Sigma$, in the context of Einstein-Maxwell-dilaton theory. We have applied Darmois-Israel formalism and the cut-and-paste method by joining together two identical space-time solutions. We carry out the dyonic thin-shell wormhole stability analyses by using a linear barotropic gas, Chaplygin gas, and logarithmic gas for the exotic matter. It is shown that, by choosing suitable parameter values as well as equation of state parameter, under specific conditions, we obtain a stable dyonic thin-shell wormhole solution. Finally, we argue that the stability domain of the dyonic thin-shell wormhole can be increased in terms of electric charge, magnetic charge, and dilaton charge.

\section{Introduction}

Wormholes are exotic objects predicted by Einstein's theory of gravity which act as a space-time tunnel by connecting two different regions of the universe. Though the idea of wormholes is not new $[1,2]$, the interest in wormholes was recently reborn by the seminal work of Morris and Thorne [3] who studied traversable wormholes. There are, however, several problematic issues related to the possible existence of wormholes; in particular, it was shown that the existence of wormholes requires the violation of energy conditions $[4,5]$. Another major problem is related to the stability analysis of wormholes. On the other hand, Visser attempted to minimize the existence of the exotic matter by constructing infinitesimally small thin-shell wormholes [6-9]. Visser's method is based on the cut-and-paste technique by joining together two identical space-time solutions and making use of the Darmois-Israel formalism [10] to compute the surface stress-energy tensor components. Finally, these results can be used to study the wormhole dynamics with the help of Lanczos equations.

This method was applied to construct a number of thinshell wormholes (TSW), including charged TSW [11, 12], TSW with a cosmological constant [13], TSW in dilaton gravity [14], TSW from the regular Hayward black hole [15], TSW in higher-dimensional Einstein-Maxwell theory $[16,17]$, rotating TSW $[18,19]$, quantum corrected TSW in Bohmian quantum mechanics [20], primordial wormholes induced from Grand Unified Theories (GUTs) [21, 22], canonical acoustic TSW, charged TSW with dilaton field, TSW with a Chaplygin gas, traversable wormholes in the anti-de Sitter space-time, TSW with a negative cosmological 
constant, wormholes in mimetic gravity, TSW from charged black string, cylindrical TSW, and many other interesting papers [23-58], while the stability analysis is investigated by different models, for example, linear perturbations [9] and specific equations of state (EoS) such as linear barotropic gas (LBG), Chaplygin gas (CG), and logarithmic gas (LogG) for the exotic matter $[14,59-62]$.

Recently, Goulart found a four-dimensional, spherically symmetric, dyonic black hole and charged wormhole solution in the low-energy effective actions of string theory or supergravity theory [63, 64]. Furthermore, in [65], a timedependent spherically symmetric black hole solution in the context of low-energy string theory was investigated. The solution found by Goulart is of particular interest since it can be written in terms of five independent parameters: the electric charge $Q$, the magnetic charge $\Sigma$, the value of the dilation of infinity $\phi_{0}$, and two integration constants, $r_{1}$ and $r_{2}$. Inspired by this work, we aim to use this solution and construct a four-dimensional TSW wormhole in the context of Einstein-Maxwell-dilaton (EMD) theory and then investigate the role of electric charge $Q$, magnetic charge $P$, and dilaton charge $\Sigma$ on the stability domain of the wormhole.

The structure of this paper is as follows. In Section 2, we review briefly the dyonic black hole solutions. In Section 3, using Visser's cut-and-paste technique, we construct a dyonic thin-shell wormhole (DTSW). In Section 4, we check the stability conditions for different types of gases such as LBG, CG, and LogG for the exotic matter. In Section 5, we comment on our results.

\section{Dyonic Black Holes in the EMD Theory}

In this part, we use the dyonic black hole solutions in the EMD theory found by Goulart [63]. Firstly, we consider the action of the EMD without a dilaton potential and without an axion:

$$
S=\int d^{4} x \sqrt{-g}\left(R-2 \partial_{\mu} \phi \partial^{\mu} \phi-W(\phi) F_{\mu \nu} F^{\mu \nu}\right)
$$

where the field strength is given by

$$
F_{\mu \nu}=\partial_{\mu} A_{\nu}-\partial_{\nu} A_{\mu} .
$$

Furthermore, for constant axion field, the bosonic sector of $S U(4)$ version of $\mathcal{N}=4$ supergravity theory is $W(\phi)=e^{-2 \phi}$ [41]. It is noted that there are five independent parameters, that is, $Q, P, \phi_{0}, r_{1}$, and $r_{2}$. Accordingly, the space-time of the general spherically symmetric solution is given by the line element [63]

$$
\begin{aligned}
d s^{2}= & -f(r) d t^{2}+\frac{1}{f(r)} d r^{2} \\
& +h(r)\left(d \theta^{2}+\sin ^{2} \theta d \varphi^{2}\right),
\end{aligned}
$$

where

$$
\begin{aligned}
& f(r)=\frac{\left(r-r_{1}\right)\left(r-r_{2}\right)}{\left(r+d_{0}\right)\left(r+d_{1}\right)}, \\
& h(r)=\left(r+d_{0}\right)\left(r+d_{1}\right),
\end{aligned}
$$

$$
\begin{aligned}
e^{2 \phi} & =e^{2 \phi_{0}} \frac{r+d_{1}}{r+d_{0}}, \\
F_{r t} & =\frac{e^{2 \phi_{0}} Q}{\left(r+d_{0}\right)^{2}}, \\
F_{\theta \varphi} & =P \sin \theta,
\end{aligned}
$$

with

$$
\begin{aligned}
& d_{0}=\frac{-\left(r_{1}+r_{2}\right) \pm \sqrt{\left(r_{1}-r_{2}\right)^{2}+8 e^{2 \phi_{0}} Q^{2}}}{2}, \\
& d_{1}=\frac{-\left(r_{1}+r_{2}\right) \pm \sqrt{\left(r_{1}-r_{2}\right)^{2}+8 e^{-2 \phi_{0} P^{2}}}}{2} .
\end{aligned}
$$

Note that the corresponding electric and magnetic charges are $Q$ and $P$, respectively. $\phi_{0}$ stands for the value of the dilaton at infinity. Furthermore, there are two integration constants, that is, $r_{1}$ and $r_{2}$. On the other hand, $d_{0}$ and $d_{1}$ are dependent constants inasmuch as they transform into each other under S-duality (i.e., $Q \leftrightarrow P$ and $\phi \rightarrow-\phi$ ). It is noted that $e^{-2 \phi}$, which is the dilaton coupling, is also invariant. Here, $r_{1}$ and $r_{2}$ are the inner and outer horizons, respectively [63].

The Hawking temperature is calculated by

$$
T=\frac{1}{4 \pi} \frac{\left(r_{2}-r_{1}\right)}{\left(r_{2}+d_{0}\right)\left(r_{2}+d_{1}\right)},
$$

and the entropy of the black hole is

$$
S=\pi\left(r_{2}+d_{0}\right)\left(r_{2}+d_{1}\right)
$$

One can also define the dilaton charge as follows:

$$
\Sigma=\frac{1}{4 \pi} \int d \Sigma^{\mu} \nabla_{\mu} \phi=\frac{\left(d_{0}-d_{1}\right)}{2}
$$

where, depending on the values of electric/magnetic charge of black hole, it can be positive or negative. Firstly, the four parameters' $\left(Q, P, \phi_{0}, M\right)$ dyonic solution is found in [37]. Here, the key point is that there is no boundary condition on $r_{1}$ and $r_{2}$ to make this dyonic black hole.

The Ricci scalar is calculated as follows:

$$
R=\frac{\left(d_{0}-d_{1}\right)^{2}\left(r-r_{1}\right)\left(r-r_{2}\right)}{2\left(r+d_{0}\right)^{3}\left(r+d_{1}\right)^{3}}
$$

The domain of $h(r) \geq 0$ is restricted with the causality. The singularity is found at $r_{S}=-d_{0}$ for $d_{0}>d_{1}$, or at $r_{S}=-d_{1}$ for $d_{1}>d_{0}$.

One of the special cases which we use to construct a DTSW when $d_{1}=-d_{0}$ is that the dilaton charge is a constant 
$d_{0}$ such as $d_{0}=\Sigma$. Furthermore, we suppose that $\left(r_{1}+r_{2}\right)=$ $2 M$ and $\left(r_{1} r_{2}\right)=r_{0}^{2}$ [37]. The solution becomes

$$
\begin{aligned}
f(r) & =\frac{\left(r-r_{1}\right)\left(r-r_{2}\right)}{\left(r^{2}-\Sigma^{2}\right)}, \\
h(r) & =\left(r^{2}-\Sigma^{2}\right) \\
e^{2 \phi} & =e^{2 \phi_{0}} \frac{r-\Sigma}{r+\Sigma}, \\
F_{r t} & =\frac{e^{2 \phi_{0}} Q}{(r+\Sigma)^{2}}, \\
F_{\theta \varphi} & =P \sin \theta .
\end{aligned}
$$

One can find the magnetically charged solutions of $[38,39]$ by using $Q=0$ and also the Schwarzschild solution by setting $P=0$.

\section{Construction of DTSW}

Let us now proceed to use the cut-and-paste technique to construct a DTSW using metric (3). Consider two spherically symmetric space-time solutions of the dyonic black hole metric in 4 dimensions and then remove from each fourdimensional manifold $M^{( \pm)}$the regions described by [9]

$$
M^{( \pm)}=\left\{r^{( \pm)} \leq a \mid a>r_{h}\right\}
$$

where $a$ is the radius of the throat of the DTSW with an important condition $a>r_{h}$. In other words, $a$ should be greater than the event horizon in order to avoid the formation of an event horizon. Next, paste these two manifolds at the boundary hypersurface given by $\Sigma^{( \pm)}=\left\{r^{( \pm)}=a, a>r_{h}\right\}$ which results with a geodesically complete manifold $M=$ $M^{+} \cup M^{-}$. According to the Darmois-Israel formalism, we can choose the coordinates on $M$ as $x^{\alpha}=(t, r, \theta, \varphi)$, while the coordinates on the induced metric $\Sigma$ are $\xi^{i}=(\tau, \theta, \varphi)$. For the parametric equation on $\Sigma$, we can write

$$
\Sigma: F(r, \tau)=r-a(\tau)=0 .
$$

Our main goal is to compare various characteristics of EMD theory and dyonic black hole, such as the surface stressenergy tensor and the basic question of stability. For this purpose, we define the dynamical induced metric on $\Sigma$ that can now be written in terms of the proper time $\tau$ on the shell, where $a=a(\tau)$, as follows:

$$
d s_{\Sigma}^{2}=-d \tau^{2}+a(\tau)^{2}\left(d \theta^{2}+\sin ^{2} \theta d \varphi^{2}\right) .
$$

The junction conditions on $\Sigma$ imply from the Lanczos equations

$$
S_{j}^{i}=-\frac{1}{8 \pi}\left(\left[K^{i}{ }_{j}\right]-\delta^{i}{ }_{j} K\right)
$$

in which $S_{j}^{i}=\operatorname{diag}\left(-\sigma, p_{\theta}, p_{\varphi}\right)$ is the energy momentum tensor on the thin shell and $K$ and $\left[K_{i j}\right]$ are defined as $K=$
$\operatorname{trace}\left[K_{i}^{i}\right]$ and $\left[K_{i j}\right]=K_{i j}{ }^{+}-K_{i j}{ }^{-}$, respectively. Furthermore, the extrinsic curvature $K^{i}{ }_{j}$ is defined by

$$
K_{i j}^{( \pm)}=-n_{\mu}^{( \pm)}\left(\frac{\partial^{2} x^{\mu}}{\partial \xi^{i} \partial \xi^{j}}+\Gamma_{\alpha \beta}^{\mu} \frac{\partial x^{\alpha}}{\partial \xi^{i}} \frac{\partial x^{\beta}}{\partial \xi^{j}}\right)_{\Sigma} .
$$

We can choose the unit vectors $n_{\mu}^{( \pm)}$, such that $n_{\mu} n^{\mu}=1$ and normal to $M^{( \pm)}$as follows:

$$
n_{\mu}^{( \pm)}= \pm\left(\left|g^{\alpha \beta} \frac{\partial F}{\partial x^{\alpha}} \frac{\partial F}{\partial x^{\beta}}\right|^{-1 / 2} \frac{\partial F}{\partial x^{\mu}}\right)_{\Sigma} .
$$

Adopting the orthonormal basis $\left\{e_{\widehat{\tau}}, e_{\hat{\theta}}, e_{\hat{\varphi}}\right\}\left(e_{\widehat{\tau}}=e_{\tau}, e_{\widehat{\theta}}=\right.$ $\left.[h(a)]^{-1 / 2} e_{\theta}, e_{\hat{\varphi}}=\left[h(a) \sin ^{2} \theta\right]^{-1 / 2} e_{\varphi}\right)$, for metric (3), the extrinsic curvature components are found as [24]

$$
\begin{aligned}
& K_{\tilde{\theta} \hat{\theta}}^{ \pm}=K_{\widehat{\varphi} \hat{\varphi}}^{ \pm}= \pm \frac{h^{\prime}(a)}{2 h(a)} \sqrt{f(a)+\dot{a}^{2}}, \\
& K_{\widehat{\tau} \hat{\tau}}^{ \pm}=\mp \frac{2 \ddot{a}+f^{\prime}(a)}{2 \sqrt{f(a)+\dot{a}^{2}}}
\end{aligned}
$$

where the prime and the dot represent the derivatives with respect to $r$ and $\tau$, respectively. With the definitions of $\left[K_{\widehat{i}}\right] \equiv$ $K_{\hat{i} j}^{+}-K_{\widehat{i} \hat{j}}^{-}$and $K=\operatorname{tr}\left[K_{\widehat{i} \hat{j}}\right]=\left[K_{\hat{i}}^{\hat{i}}\right]$ and the introduction of the surface stress-energy tensor $S_{\widehat{i} j}=\operatorname{diag}\left(\sigma, p_{\widehat{\theta}}, p_{\widehat{\varphi}}\right)$, we have the Einstein equations on the shell (also called the Lanczos equations):

$$
-\left[K_{\overparen{i} \mathfrak{j}}\right]+K g_{\widehat{i} \hat{j}}=8 \pi S_{\widehat{i} \hat{j}},
$$

which in our case results in a shell of radius $a$ with energy density $\sigma$ and transverse pressure $p=p_{\hat{\theta}}=p_{\hat{\varphi}}$. Using the above results from the Lanczos equations, one can easily check that the surface density and the surface pressure are given by the following relations $[24,25]$ :

$$
\begin{aligned}
& \sigma=-\frac{\sqrt{f(a)+\dot{a}^{2}}}{4 \pi} \frac{h^{\prime}(a)}{h(a)}, \\
& p=\frac{\sqrt{f(a)+\dot{a}^{2}}}{8 \pi}\left[\frac{2 \ddot{a}+f^{\prime}(a)}{f(a)+\dot{a}^{2}}+\frac{h^{\prime}(a)}{h(a)}\right] .
\end{aligned}
$$

Note that the energy density is negative at the throat because of the flare-out condition in which the area is minimal at the throat (then $h(r)$ increases for $r$ close to $a$ and $h^{\prime}(a)>0$ ), so we have exotic matter. From the last two equations, we can now write the static configuration of radius $a$; by setting $\dot{a}=0$ and $\ddot{a}=0$, we get

$$
\begin{aligned}
& \sigma_{0}=-\frac{\sqrt{f\left(a_{0}\right)}}{4 \pi} \frac{h^{\prime}\left(a_{0}\right)}{h\left(a_{0}\right)}, \\
& p_{0}=\frac{\sqrt{f\left(a_{0}\right)}}{8 \pi}\left[\frac{f^{\prime}\left(a_{0}\right)}{f\left(a_{0}\right)}+\frac{h^{\prime}\left(a_{0}\right)}{h\left(a_{0}\right)}\right] .
\end{aligned}
$$


From (21), we see that the surface density is negative (i.e., $\left.\sigma_{0}<0\right)$; as a consequence of this, the WEC is violated. The amount of exotic matter concentrated at the wormhole is calculated by the following integral:

$$
\Omega_{\sigma}=\int \sqrt{-g}\left(\rho+p_{r}\right) d^{3} x
$$

In the case of a TSW, we have $p_{r}=0$ and $\rho=\sigma \delta(r-a)$, where $\delta(r-a)$ is the Dirac delta function. The above integral can be easily evaluated if we first make use of the Dirac delta function

$$
\Omega_{\sigma}=\int_{0}^{2 \pi} \int_{0}^{\pi} \int_{-\infty}^{\infty} \sigma \sqrt{-g} \delta(r-a) d r d \theta d \varphi .
$$

Substituting the value of energy density in the last equation, for the energy density located on a thin-shell surface in static configuration, we find

$$
\Omega_{\sigma}=-2 a_{0} \sqrt{\frac{\left(a_{0}-r_{1}\right)\left(a_{0}-r_{2}\right)}{\left(a_{0}^{2}-\Sigma^{2}\right)}} .
$$

To analyze the attractive and repulsive nature of the wormhole, we can calculate the observer's four-acceleration $a^{\mu}=u^{\nu} \nabla_{\nu} u^{\mu}$, where the four-velocity reads $u^{\mu}=(1 / \sqrt{f(r)}$, $0,0,0)$. For the radial component of the four-acceleration, we find

$$
\begin{aligned}
a^{r} & =\Gamma_{t t}^{r}\left(\frac{d t}{d \tau}\right)^{2} \\
& =\frac{a_{0}^{2}\left(r_{1}+r_{2}\right)-2 a_{0}\left(\Sigma^{2}+r_{1} r_{2}\right)+\Sigma^{2}\left(r_{1}+r_{2}\right)}{2\left(a_{0}^{2}-\Sigma^{2}\right)^{2}} .
\end{aligned}
$$

One can easily observe that the test particle obeys the equation of motion

$$
\frac{d^{2} r}{d \tau^{2}}=-\Gamma_{t t}^{r}\left(\frac{d t}{d \tau}\right)^{2}=-a^{r}
$$

We conclude from the last equation that if $a^{r}=0$, we get the geodesic equation, while the wormhole is attractive when $a^{r}>0$ and repulsive when $a^{r}<0$.

\section{Stability Analysis}

In this section, using the formalism developed in Section 3, we calculate the potential and define the stability method for DTSW. From the energy conservation, we have [24]

$$
\begin{aligned}
\frac{d}{d \tau} & (\sigma \mathscr{A})+p \frac{d \mathscr{A}}{d \tau} \\
& =\left\{\left[h^{\prime}(a)\right]^{2}-2 h(a) h^{\prime \prime}(a)\right\} \frac{\dot{a} \sqrt{f(a)+\dot{a}^{2}}}{2 h(a)},
\end{aligned}
$$

where the area of the wormhole is calculated by $\mathscr{A}=4 \pi h(a)$. It is noted that the internal energy of the throat is located at the left side of (28) as a first term. Then, the second term represents the work done by the internal forces of the throat; on the other hand, there is a flux term in the right side of the equation. Furthermore, to calculate the equation of dynamics of the wormhole, we use $\sigma(a)$ in (19) and find this simple equation:

$$
\dot{a}^{2}=-V(a)
$$

with potential

$$
V(a)=f(a)-16 \pi^{2}\left[\frac{h(a)}{h^{\prime}(a)} \sigma(a)\right]^{2} .
$$

A Taylor expansion to the second order of the potential $V(a)$ around the static solution yields [24]

$$
\begin{aligned}
V(a)= & V\left(a_{0}\right)+V^{\prime}\left(a_{0}\right)\left(a-a_{0}\right)+\frac{V^{\prime \prime}\left(a_{0}\right)}{2}\left(a-a_{0}\right)^{2} \\
& +O\left(a-a_{0}\right)^{3} .
\end{aligned}
$$

From (30), the first derivative of $V(a)$ is

$$
\begin{aligned}
& V^{\prime}(a)=f^{\prime}(a)-32 \pi^{2} \sigma(a) \\
& \cdot \frac{h(a)}{h^{\prime}(a)}\left\{\left[1-\frac{h(a) h^{\prime \prime}(a)}{\left[h^{\prime}(a)\right]^{2}}\right] \sigma(a)\right. \\
& \left.+\frac{h(a)}{h^{\prime}(a)} \sigma^{\prime}(a)\right\},
\end{aligned}
$$

and the last equation takes the form

$$
V^{\prime}(a)=f^{\prime}(a)+16 \pi^{2} \sigma(a) \frac{h(a)}{h^{\prime}(a)}[\sigma(a)+2 p(a)] .
$$

The second derivative of the potential is

$$
\begin{aligned}
& V^{\prime \prime}(a)=f^{\prime \prime}(a)+16 \pi^{2}\left\{\left[\frac{h(a)}{h^{\prime}(a)} \sigma^{\prime}(a)\right.\right. \\
& \left.+\left(1-\frac{h(a) h^{\prime \prime}(a)}{\left[h^{\prime}(a)\right]^{2}}\right) \sigma(a)\right][\sigma(a)+2 p(a)] \\
& \left.+\frac{h(a)}{h^{\prime}(a)} \sigma(a)\left[\sigma^{\prime}(a)+2 p^{\prime}(a)\right]\right\} .
\end{aligned}
$$

Since $\sigma^{\prime}(a)+2 p^{\prime}(a)=\sigma^{\prime}(a)\left[1+2 p^{\prime}(a) / \sigma^{\prime}(a)\right]$, replacing the parameter $p=\psi(\sigma)$ and $\psi^{\prime}=d p / d \sigma=p^{\prime} / \sigma^{\prime}$, we have that $\sigma^{\prime}(a)+2 p^{\prime}(a)=\sigma^{\prime}(a)\left(1+2 \psi^{\prime}\right)$, and using (35) again, we obtain

$$
\begin{aligned}
& V^{\prime \prime}\left(a_{0}\right)=f^{\prime \prime}\left(a_{0}\right)-8 \pi^{2}\left\{\left[\sigma_{0}+2 p_{0}\right]^{2}\right. \\
& +2 \sigma_{0}\left[\left(\frac{3}{2}-\frac{h\left(a_{0}\right) h^{\prime \prime}\left(a_{0}\right)}{\left[h^{\prime}\left(a_{0}\right)\right]^{2}}\right) \sigma_{0}+p_{0}\right] \\
& \left.\cdot\left(1+2 \psi^{\prime}\right)\right\} .
\end{aligned}
$$

The wormhole is stable if and only if $V^{\prime \prime}\left(a_{0}\right)>0$. 


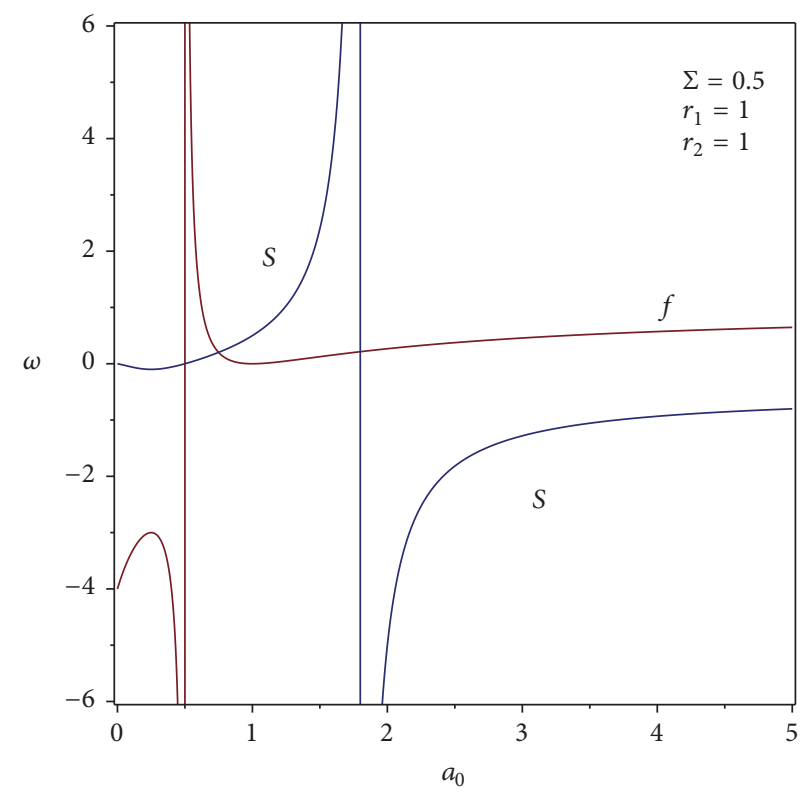

(a)

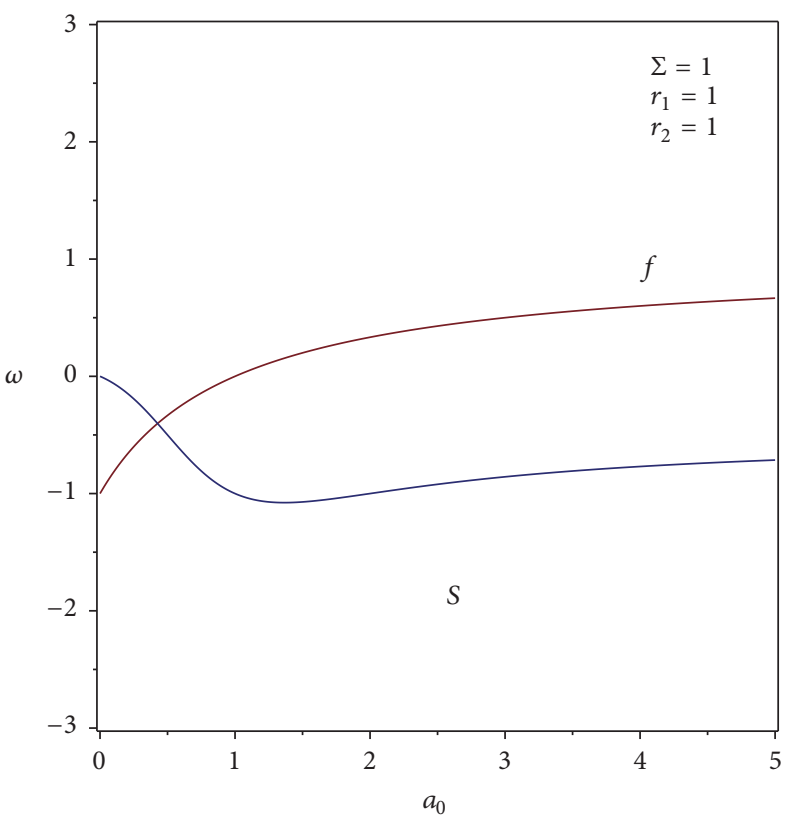

(c)

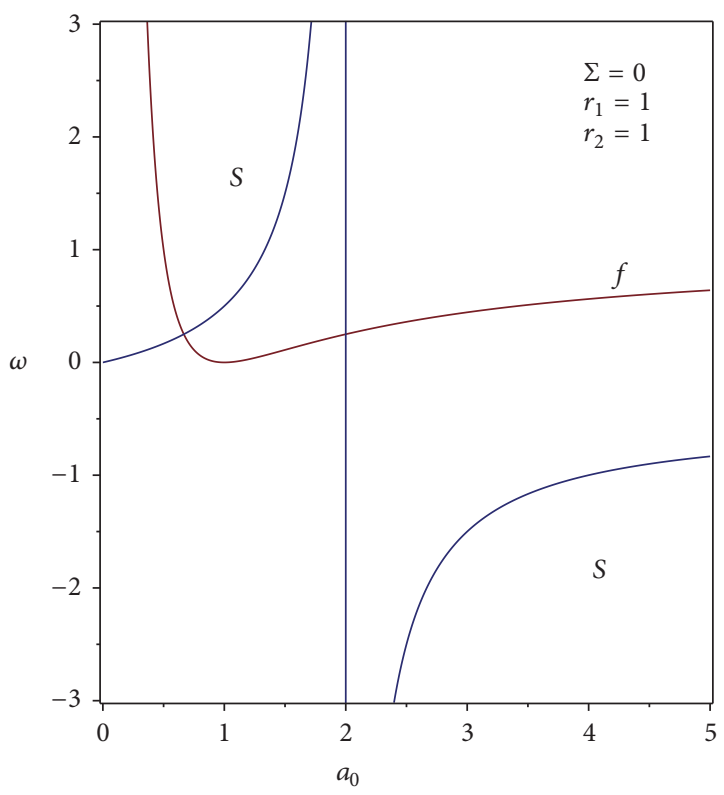

(b)

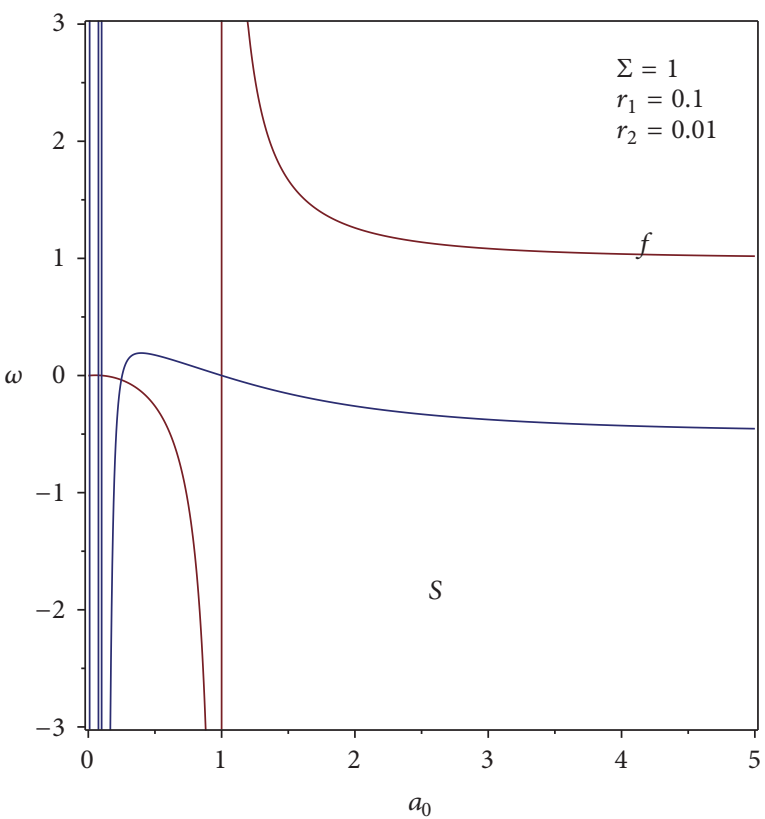

(d)

FIGURE 1: Stability regions of DTSW in terms of $\omega$ and radius of the throat $a_{0}$ for different values of $\Sigma, r_{1}$, and $r_{2}$.

4.1. Stability Analysis of DTSW via the LBG. In what follows, we will use three different gas models for the exotic matter to explore the stability analysis: LBG [40], CG [61, 62], and finally $\log G$ [15].

The equation of state of $\operatorname{LBG}[9,14,59,60]$ is given by

$$
\psi=\omega \sigma,
$$

and hence

$$
\psi^{\prime}\left(\sigma_{0}\right)=\omega
$$

where $\omega$ is a constant parameter. For more useful information as regards the effects of the parameters $\Sigma, r_{1}$, and $r_{2}$, we show graphically the DTSW stability in terms of $\omega$ and $a_{0}$, as depicted in Figure 1.

By changing the values of $\Sigma, r_{1}$, and $r_{2}$, which encode the effects of electric $Q$, magnetic $P$, and dilaton charge $\phi_{0}$ on the DTSW stability, we see from Figure 1 that in two cases the region of stability is below the curve in the interval to the right of the asymptote, while in two other cases the stability region is simply below the curve. The region of stability is denoted by $S$.

4.2. Stability Analysis of DTSW via CG. The equation of state of CG that we considered is given by [61] 

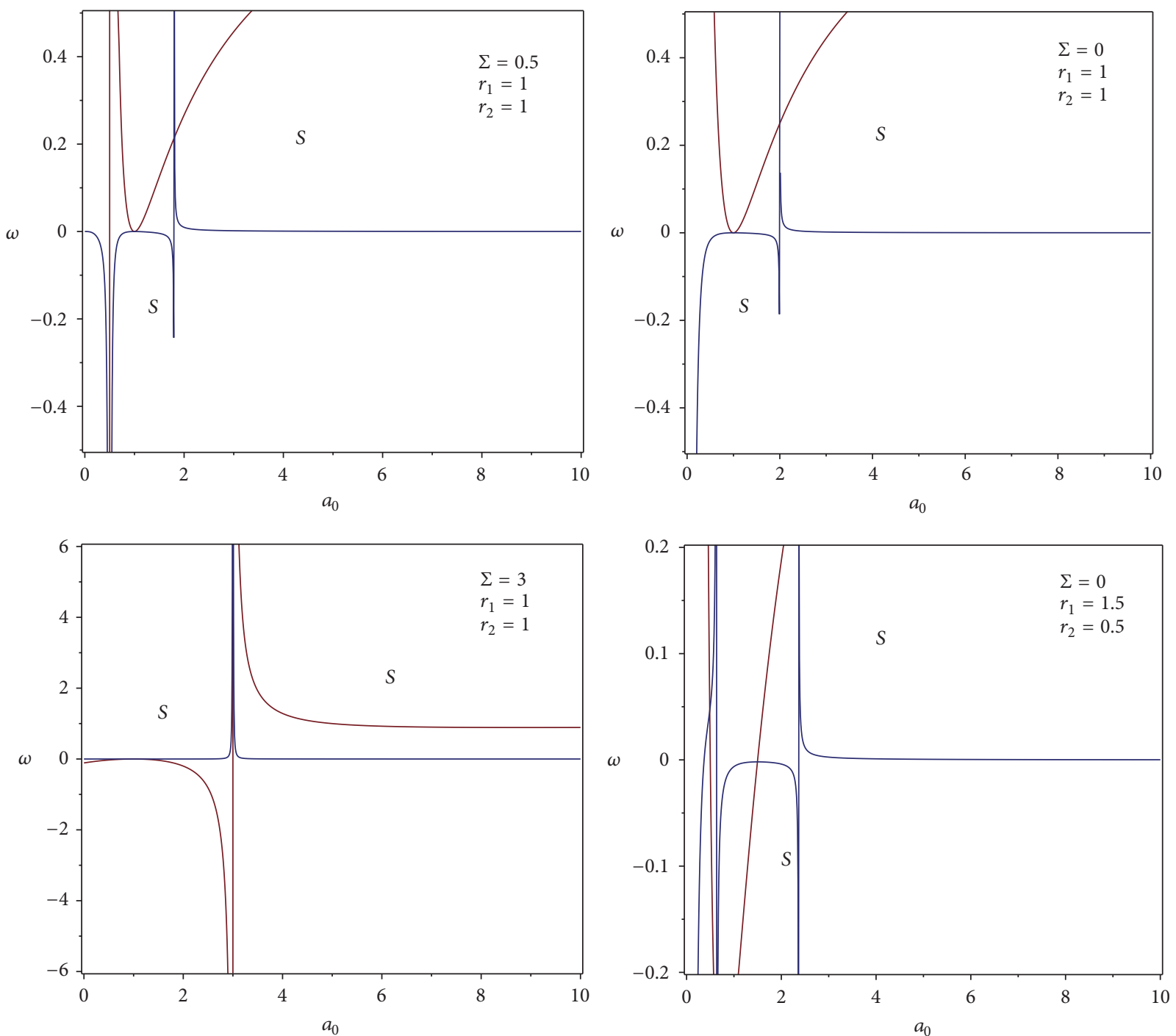

FIGURE 2: Stability regions of DTSW in terms of $\omega$ as a function of the throat $a_{0}$ for different values of $\Sigma, r_{1}$, and $r_{2}$.

$$
\psi=\omega\left(\frac{1}{\sigma}-\frac{1}{\sigma_{0}}\right)+p_{0},
$$

and one naturally finds

$$
\psi^{\prime}\left(\sigma_{0}\right)=-\frac{\omega}{\sigma_{0}^{2}} .
$$

After inserting (38) into (29), we plot the stability regions of DTSW supported by CG in terms of $V^{\prime \prime}\left(a_{0}\right)$ and $a_{0}$ as shown in Figure 2. It is worth mentioning that in three cases the region of stability is above the curve in the interval to the right of the asymptote, while in one case stability region is below the curve in the interval to the right of the asymptote. The region of stability is denoted by $S$.

4.3. Stability Analysis of DTSW via LogG. In our final example, the equation of state for LogG is selected as follows [15]:

$$
\psi=\omega \ln \left(\frac{\sigma}{\sigma_{0}}\right)+p_{0}
$$

which leads to

$$
\psi^{\prime}\left(\sigma_{0}\right)=\frac{\omega}{\sigma_{0}}
$$

After inserting the above expression into (29), we show the stability regions of TSW supported by LogG in Figure 3. In this case, we see that the region of stability is above the curve in the interval to the right of the asymptote. The region of stability is denoted by $S$.

\section{Conclusion}

In this work, we have constructed a stable DTSW in the context of EMD theory. In particular, we explore the role of three parameters $-\Sigma, r_{1}$, and $r_{2}$ - which encode the effects of electric charge $Q$, magnetic charge $P$, and dilaton charge $\phi_{0}$ on the wormhole stability. The surface stress at the wormhole throat is computed via Darmois-Israel formalism while the stability analyses are carried out by using three different 

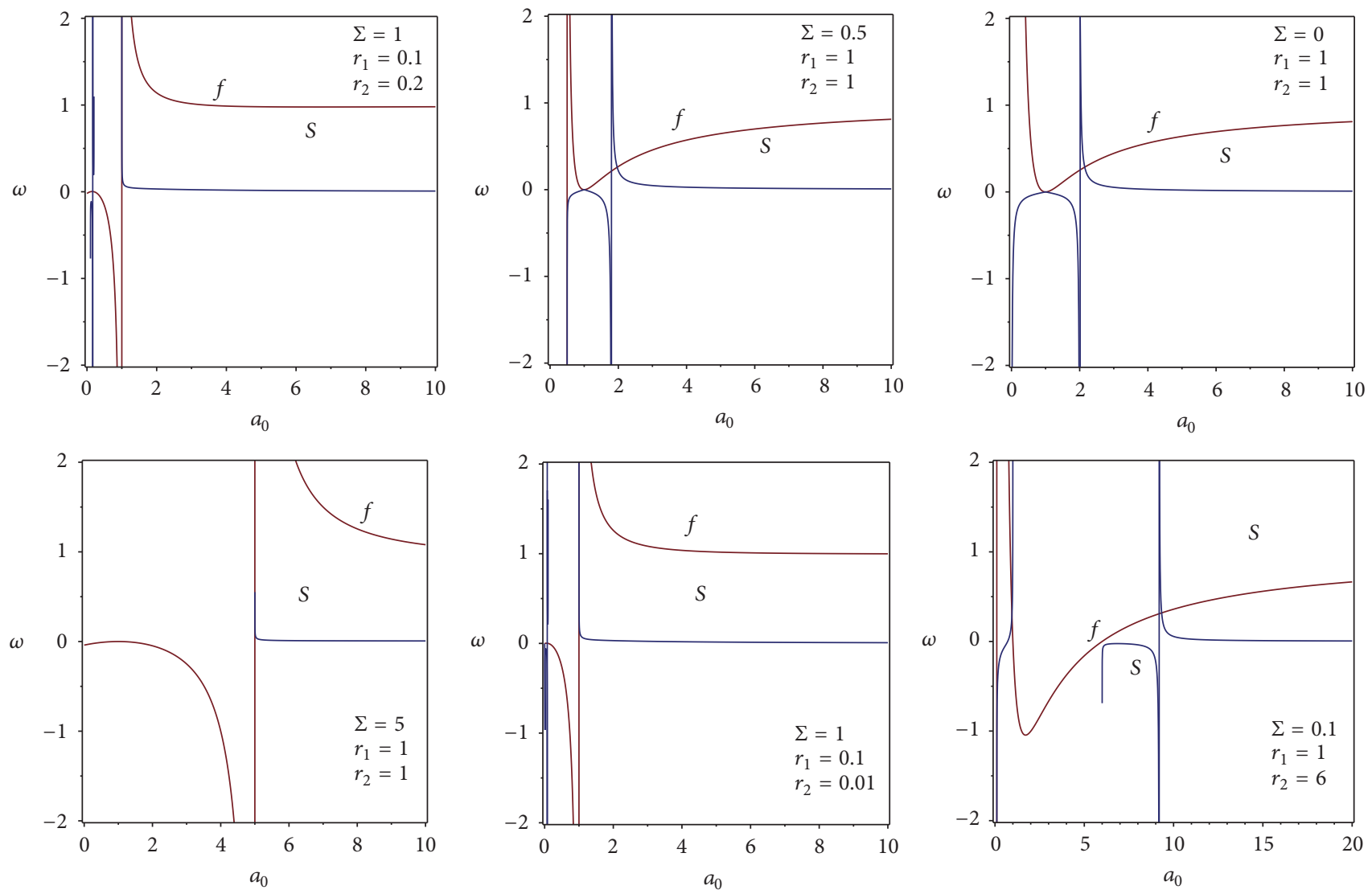

FIGURE 3: Stability regions of DTSW in terms of $\omega$ and radius of the throat $a_{0}$ for different values of $\Sigma, r_{1}$, and $r_{2}$.

models. As a first model, we consider LBG and show that the wormhole can be stable by choosing suitable values of parameters $\Sigma, r_{1}$, and $r_{2}$. In the second case, we focus on the stability analyses using a CG for the exotic matter and show this by choosing suitable values of parameters $\Sigma$, $r_{1}$, and $r_{2}$. Finally, we use LogG for the exotic matter and show similar results. The results show that electric charge, magnetic charge, and dilaton charge play an important role in DTSW by increasing the stability domain of the wormhole. A particularly interesting finding is that, for suitable values of $a_{0}$, the stable solutions exist in the DTSW for each value of $\omega$ that is chosen. We conclude that DTSW is linearly stable for variable EoS, which supports the fact that the presence of EoS and dilaton/electric/magnetic charges induces stability in the WH geometry. In particular, we can see from Figures 1(a) and 1(b) that, by keeping $r_{1}$ and $r_{2}$ fixed and changing $\Sigma$, the vertical asymptote is slightly shifted to the left with the increase of $\Sigma$. This clearly indicates the increase of the stability region in the right of the asymptote. On the other hand, another model of charged TSW was constructed by Eiroa and Simeone [14] in low-energy string gravity, which supports our results. Their results show $\rho$ and $p$ on the shell for the null dilaton coupling parameter and it is shown that exotic matter is localized; moreover, they managed to minimize the exotic matter needed using the stronger dilaton-Maxwell coupling.

\section{Conflicts of Interest}

The authors declare that there are no conflicts of interest regarding the publication of this paper.

\section{Acknowledgments}

This work was supported by the Chilean FONDECYT Grant no. 3170035 (Ali Övgün).

\section{References}

[1] L. Flamm, "Beiträge zur Einsteinschen Gravitationstheorie," Physikalische Zeitschrift, vol. 17, pp. 484-454, 1916.

[2] A. Einstein and N. Rosen, "The particle problem in the general theory of relativity," Physical Review D: Particles, Fields, Gravitation and Cosmology, vol. 48, no. 1, pp. 73-77, 1935.

[3] M. S. Morris and K. S. Thorne, "Wormholes in spacetime and their use for interstellar travel: a tool for teaching general relativity," American Journal of Physics, vol. 56, no. 5, pp. 395-412, 1988.

[4] M. S. Morris, K. S. Thorne, and U. Yurtsever, "Wormholes, time machines, and the weak energy condition," Physical Review Letters, vol. 61, no. 13, pp. 1446-1449, 1988.

[5] A. Övgün and M. Halilsoy, "Existence of traversable wormholes in the spherical stellar systems," Astrophysics and Space Science, vol. 361, no. 7, article no. 214, 2016. 
[6] M. Visser, Lorentzian Wormholes, AIP Press, New York, NY, USA, 1996.

[7] M. Visser, "Traversable wormholes from surgically modified Schwarzschild spacetimes," Nuclear Physics. B. Theoretical, Phenomenological, and Experimental High Energy Physics. Quantum Field Theory and Statistical Systems, vol. 328, no. 1, pp. 203212, 1989.

[8] M. Visser, "Traversable wormholes: some simple examples," Physical Review D: Particles, Fields, Gravitation and Cosmology, vol. 39, no. 10, pp. 3182-3184, 1989.

[9] E. Poisson and M. Visser, "Thin-shell wormholes: linearization stability," Physical Review D: Particles, Fields, Gravitation and Cosmology, vol. 52, no. 12, pp. 7318-7321, 1995.

[10] W. Israel, "Singular hypersurfaces and thin shells in general relativity," Il Nuovo Cimento B, vol. 44, no. 1, pp. 1-14, 1966.

[11] E. F. Eiroa and G. E. Romero, "Linearized stability of charged thin-shell wormholes," General Relativity and Gravitation, vol. 36, no. 4, pp. 651-659, 2004.

[12] A. Banerjee, "Stability of charged thin-shell wormholes in $(2+1)$ dimensions," International Journal of Theoretical Physics, vol. 52, no. 8, pp. 2943-2958, 2013.

[13] F. S. N. Lobo and P. Crawford, "Linearized stability analysis of thin-shell wormholes with a cosmological constant," Classical and Quantum Gravity, vol. 21, no. 2, pp. 391-404, 2004.

[14] E. F. Eiroa and C. Simeone, "Thin-shell wormholes in dilaton gravity," Physical Review D: Particles, Fields, Gravitation and Cosmology, vol. 71, no. 12, Article ID 127501, 2005.

[15] M. Halilsoy, A. Ovgun, and S. H. Mazharimousavi, "Thin-shell wormholes from the regular Hayward black hole," The European Physical Journal C, vol. 74, no. 3, pp. 1-7, 2014.

[16] F. Rahaman, M. Kalam, and S. Chakraborty, "Thin shell wormholes in higher dimensional Einstein-Maxwell theory," General Relativity and Gravitation, vol. 38, no. 11, pp. 1687-1695, 2006.

[17] F. Rahaman, P. K. Kuhfittig, M. Kalam, A. A. Usmani, and S. Ray, "A comparison of Horava-Lifshitz gravity and Einstein gravity through thin-shell wormhole construction," Classical and Quantum Gravity, vol. 28, no. 15, Article ID 155021, 2011.

[18] S. H. Mazharimousavi and M. Halilsoy, "Counter-rotational effects on stability of $2+1$-dimensional thin-shell wormholes," The European Physical Journal C, vol. 74, no. 9, article 3073, 2014.

[19] A. Ovgun, "Rotating thin-shell wormhole," The European Physical Journal Plus, vol. 131, no. 11, article no. 389, 2016.

[20] K. Jusufi, "Quantum corrected Schwarzschild thin-shell wormhole," The European Physical Journal C, vol. 76, no. 11, article no. 608, 2016.

[21] S. Nojiri, O. Obregon, S. Odintsov, and K. Osetrin, "Can primordial wormholes be induced by GUTs at the early Universe?" Physics Letters B, vol. 458, no. 1, pp. 19-28, 1999.

[22] S. Nojiri, O. Obregón, S. D. Odintsov, and K. E. Osetrin, "Induced wormholes due to quantum effects of spherically reduced matter in large $N$ approximation," Physics Letters B, vol. 449, no. 3-4, pp. 173-179, 1999.

[23] K. Jusufi and A. Övgün, "Canonical acoustic thin-shell wormholes," Modern Physics Letters A, vol. 32, no. 7, Article ID 1750047, 2017.

[24] E. F. Eiroa, "Stability of thin-shell wormholes with spherical symmetry," Physical Review D: Particles, Fields, Gravitation and Cosmology, vol. 78, no. 2, Article ID 024018, 2008.

[25] E. F. Eiroa, "Thin-shell wormholes with a generalized Chaplygin gas," Physical Review D: Particles, Fields, Gravitation and Cosmology, vol. 80, Article ID 044033, 2009.
[26] J. P. S. Lemos and F. S. N. Lobo, "Plane symmetric traversable wormholes in an anti-de Sitter background," Physical Review $D$, vol. 69, Article ID 104007, 2004.

[27] J. P. Lemos and F. S. Lobo, "Plane symmetric thin-shell wormholes: solutions and stability," Physical Review D: Particles, Fields, Gravitation and Cosmology, vol. 78, no. 4, Article ID 044030, 9 pages, 2008.

[28] F. Rahaman, A. Banerjee, and I. Radinschi, "A new class of stable $(2+1)$ dimensional thin shell wormhole," International Journal of Theoretical Physics, vol. 51, no. 6, pp. 1680-1691, 2012.

[29] R. Myrzakulov, L. Sebastiani, S. Vagnozzi, and S. Zerbini, "Static spherically symmetric solutions in mimetic gravity: rotation curves and wormholes," Classical and Quantum Gravity, vol. 33, no. 12, Article ID 125005, 21 pages, 2016.

[30] S.-W. Kim, H.-j. Lee, S. K. Kim, and J. Yang, "(2+1)-dimensional Schwarzschild-de SITter wormhole," Physics Letters A, vol. 183, no. 5-6, pp. 359-362, 1993.

[31] P. K. F. Kuhfittig, "The stability of thin-shell wormholes with a phantom-like equation of state," Acta Physica Polonica B, vol. 41, no. 9, pp. 2017-2029, 2010.

[32] M. Sharif and M. Azam, "Stability analysis of thin-shell wormholes from charged black string," Journal of Cosmology and Astroparticle Physics, vol. 2013, no. 4, article 023, 2013.

[33] M. Sharif and M. Azam, "Mechanical stability of cylindrical thin-shell wormholes," The European Physical Journal C, vol. 73, article 2407, 2013.

[34] M. Sharif and S. Mumtaz, "Dynamics of thin-shell wormholes with different cosmological models," International Journal of Modern Physics D: Gravitation, Astrophysics, Cosmology, vol. 26, no. 5, Article ID 1741007, 14 pages, 2017.

[35] M. Sharif and S. Mumtaz, "Influence of nonlinear electrodynamics on stability of thin-shell wormholes," Astrophysics and Space Science, vol. 361, no. 7, article 218, 2016.

[36] M. Sharif and F. Javed, "On the stability of bardeen thin-shell wormholes," General Relativity and Gravitation, vol. 48, no. 12, article 158, 2016.

[37] R. Kallosh, A. Linde, T. Ortín, A. Peet, and A. Van Proeyen, "Supersymmetry as a cosmic censor," Physical Review D: Particles, Fields, Gravitation and Cosmology, vol. 46, no. 12, pp. 52785302, 1992.

[38] G. W. Gibbons and K.-i. Maeda, "Black holes and membranes in higher-dimensional theories with dilaton fields," Nuclear Physics. B. Theoretical, Phenomenological, and Experimental High Energy Physics. Quantum Field Theory and Statistical Systems, vol. 298, no. 4, pp. 741-775, 1988.

[39] D. Garfinkle, G. T. Horowitz, and A. Strominger, "Charged black holes in string theory," Physical Review D, vol. 43, p. 3140, 1991, Erratum in Physical Review D, vol. 45, no. 10, p. 3888, 1992.

[40] P. K. Kuhfittig, "Wormholes with a barotropic equation of state admitting a one-parameter group of conformal motions," Annals of Physics, vol. 355, pp. 115-120, 2015.

[41] E. Cremmer, J. Scherk, and S. Ferrara, "Su(4) invariant supergravity theory," Physics Letters B, vol. 74, no. 1-2, pp. 61-64, 1978.

[42] M. Zaeem-ul-Haq Bhatti, Z. Yousaf, and S. Ashraf, "Charged black string thin-shell wormholes in modified gravity," Annals of Physics, vol. 383, pp. 439-454, 2017.

[43] A. Övgün and K. Jusufi, "Stability of effective thin-shell wormholes under lorentz symmetry breaking supported by dark matter and dark energy," https://arxiv.org/abs/1706.07656.

[44] M. Zaeem-ul-Haq Bhatti, A. Anwar, and S. Ashraf, "Construction of thin shell wormholes from metric $\mathrm{f}(\mathrm{R})$ gravity," Modern Physics Letters A, vol. 32, no. 20, Article ID 1750111, 2017. 
[45] F. S. N. Lobo, "Wormhole basics," in Wormholes, Warp Drives and Energy Conditions, vol. 189 of Fundamental Theories of Physics, pp. 11-34, Springer, Cham, Germany, 2017.

[46] D. Wang and X. H. Meng, "Thin-shell wormholes constrained by cosmological observations," Physics of the Dark Universe, vol. 17, pp. 46-51, 2017.

[47] A. Eid, "Stability of thin shell wormholes in Born-Infeld theory supported by polytropic phantom energy," The Korean Physical Society, vol. 70, no. 4, pp. 436-441, 2017.

[48] S. Chakraborty, General Relativity and Gravitation, vol. 49, no. 3, p. 47, 2017.

[49] A. Övgün and I. G. Salako, "Thin-shell wormholes in neoNewtonian theory," Modern Physics Letters A, vol. 32, no. 23, Article ID 1750119, 14 pages, 2017.

[50] A. Banerjee, K. Jusufi, and S. Bahamonde, "Stability of a $d$ dimensional thin-shell wormhole surrounded by quintessence," https://arxiv.org/abs/1612.06892.

[51] E. Guendelman, E. Nissimov, S. Pacheva, and M. Stoilov, Bulg. J. Phys, vol. 44, p. 85, 2017.

[52] EiroaE. F. and G. F. Aguirre, "Thin-shell wormholes with a double layer in quadratic F(R) gravity," Physical Review D, vol. 94, no. 4, Article ID 044016, 2016.

[53] M. Sharif and S. Mumtaz, "Stability of thin-shell wormholes from a regular ABG black hole," The European Physical Journal Plus, vol. 132, no. 1, p. 26, 2017.

[54] M. Azam, "Born-Infeld thin-shell wormholes supported by generalized cosmic Chaplygin gas," Astrophysics and Space Science, vol. 361, no. 3, article 96, 2016.

[55] A. Eid, "On the stability of charged thin-shell wormholes," The European Physical Journal Plus, vol. 131, no. 2, article 23, 2016.

[56] M. Sharif and S. Mumtaz, "Stability of the regular hayward thinshell wormholes," Advances in High Energy Physics, vol. 2016, Article ID 2868750, 13 pages, 2016.

[57] E. F. Eiroa and G. F. Aguirre, "Thin-shell wormholes with charge in $\mathrm{F}(\mathrm{R})$ gravity," The European Physical Journal C, vol. 76, no. 3, article no. 132, 2016.

[58] M. Sharif and S. Mumtaz, "Schwarzschild-de sitter and anti-de sitter thin-shell wormholes and their stability," Advances in High Energy Physics, vol. 2014, Article ID 639759, 13 pages, 2014.

[59] A. Övgün and I. Sakalli, "A particular thin-shell wormhole," Theoretical and Mathematical Physics, vol. 190, no. 1, pp. 120129, 2017.

[60] V. Varela, "Note on linearized stability of Schwarzschild thinshell wormholes with variable equations of state," Physical Review D: Particles, Fields, Gravitation and Cosmology, vol. 92, no. 4, Article ID 044002, 11 pages, 2015.

[61] E. F. Eiroa and C. Simeone, "Stability of Chaplygin gas thin-shell wormholes," Physical Review D: Particles, Fields, Gravitation and Cosmology, vol. 76, no. 2, Article ID 024021, 2007.

[62] F. S. N. Lobo, "Chaplygin traversable wormholes," Physical Review D: Particles, Fields, Gravitation and Cosmology, vol. 73, no. 6, Article ID 064028, 9 pages, 2006.

[63] P. Goulart, "Dyonic black holes and dilaton charge in string theory," https://arxiv.org/abs/1611.03093.

[64] P. Goulart, "Massless black holes and charged wormholes in string theory," https://arxiv.org/abs/1611.03164.

[65] P. Aniceto and J. V. Rocha, "Dynamical black holes in lowenergy string theory," Journal of High Energy Physics, vol. 2017, article 35, 2017. 

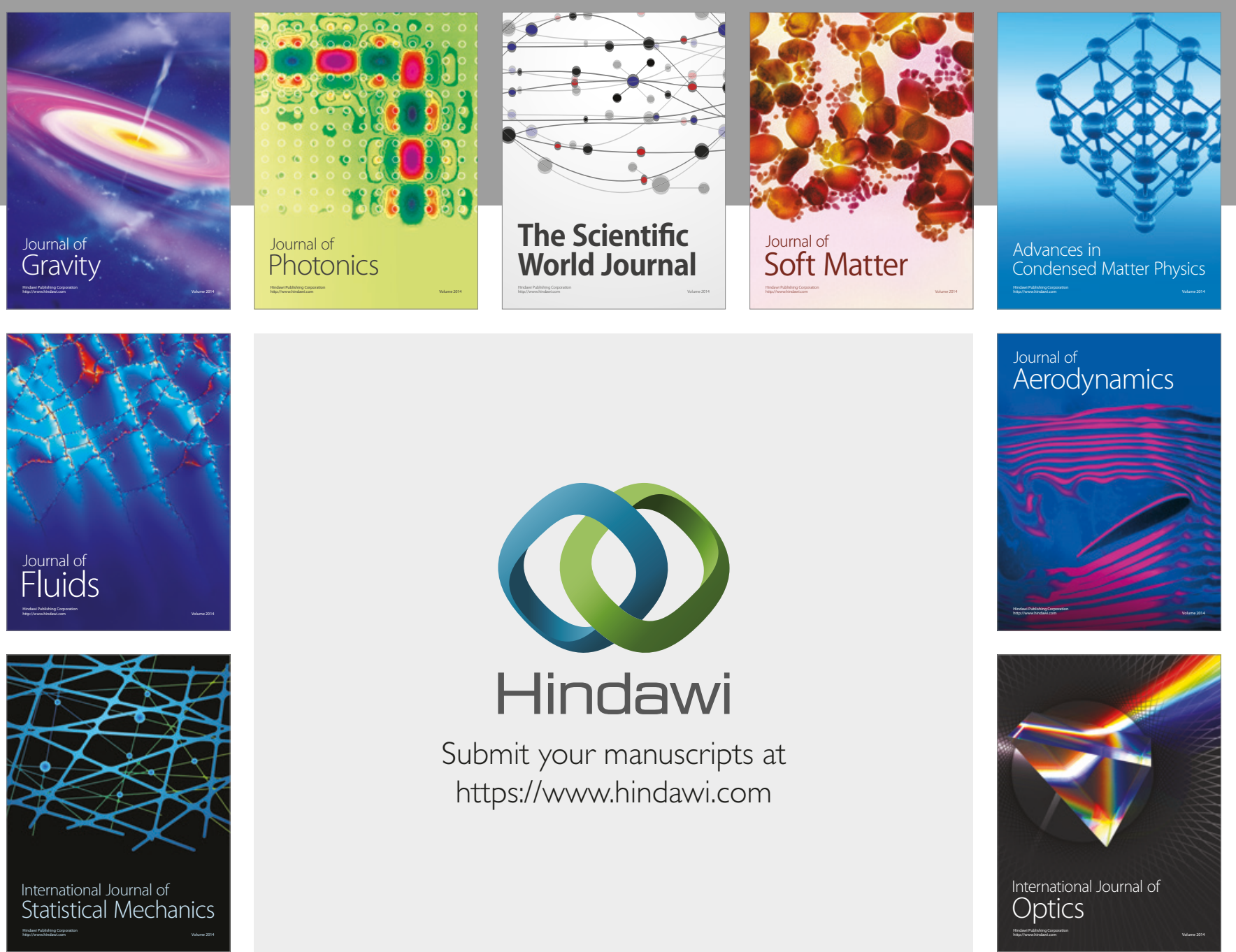

Submit your manuscripts at

https://www.hindawi.com
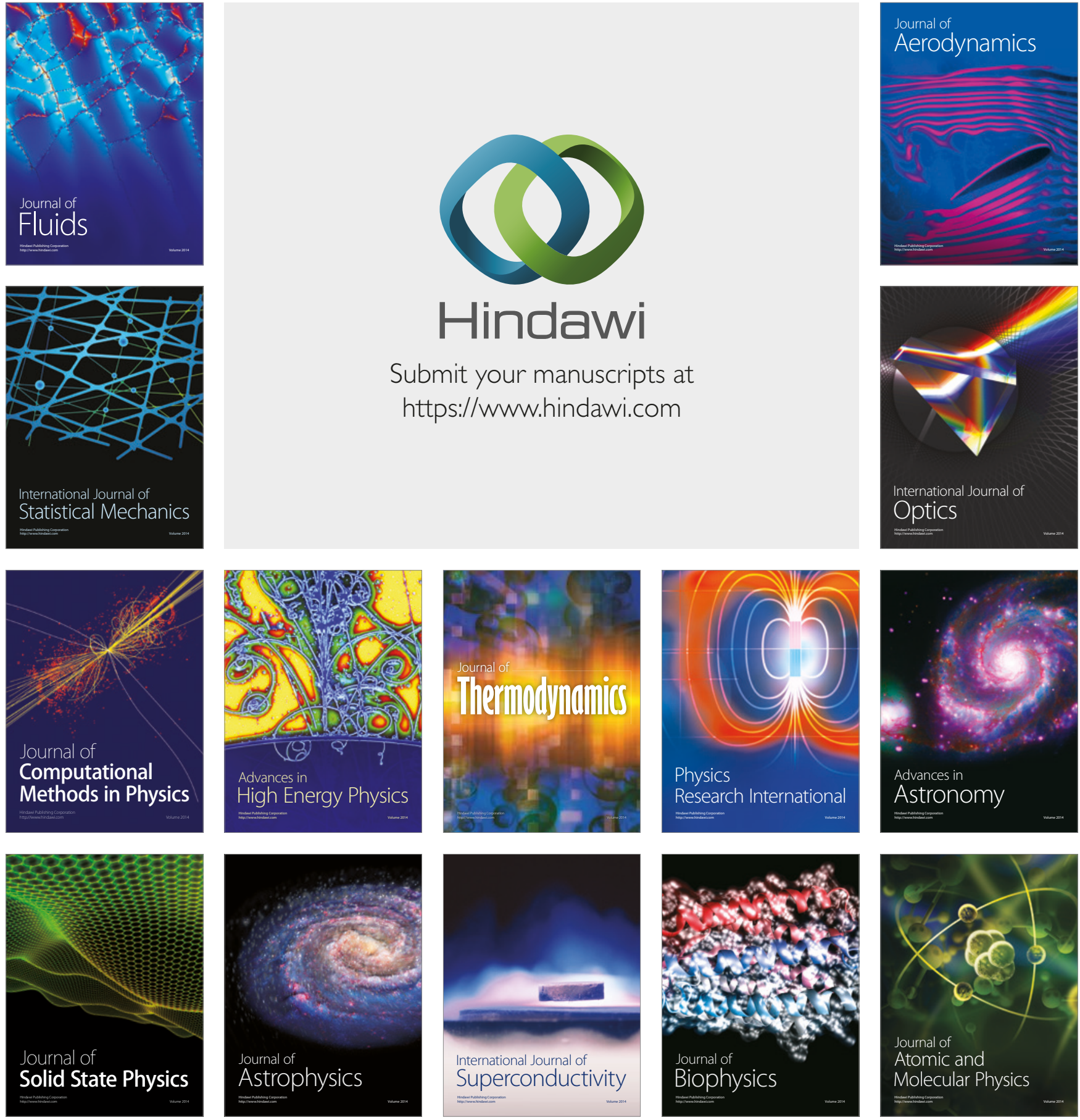